\title{
EFECTO DE DIFERENTES ANTICONTAMINANTES EN LA DIETA ARTIFICIAL EN LA CRÍA DE BROCA DEL CAFÉ
}

\section{EFFECT OF DIFFERENT ANTIMICROBIALS ON THE ARTIFICIAL DIET FOR COFFEE BERRY BORER REARING}

\author{
Tito Bacca $^{1 *}$ y Pablo Benavides ${ }^{2}$ \\ Recibido para publicación: Septiembre 28 de 2013 - Aceptado para publicación: Abril 7 de 2014
}

\begin{abstract}
RESUMEN
El uso de anticontaminantes en las dietas artificiales para la cría masiva de insectos, son comúnmente utilizados para evitar la proliferación de microorganismos del ambiente, impidiendo la contaminación del alimento y espacio de los insectos criados. Por lo tanto, el objetivo de esta investigación fue evaluar el uso de los anticontaminantes formaldehido y nipagin en la dieta artificial Cenibroca para la cría de broca del café. Mediante este experimento, con la dieta Cenibroca, se obtuvo el mayor número de estados biológicos de broca por cada $\mathrm{cm}^{3}$ de dieta, sin embargo se consiguió el mayor porcentaje de contaminación por los hongos Aspergillus sp. y Penicillum sp. Cuando se adicionó 2,65 mL de formaldehido por litro de Cenibroca, se obtuvo la menor contaminación de las dietas (3,3\%). Esta adición no afectó la producción de estados biológicos de broca, en comparación con el resto de dietas, donde se utilizaron otros anticontaminantes. A partir de estos resultados es posible utilizar la dieta Cenibroca para cría de broca del café para la producción masiva de sus parasitoides.
\end{abstract}

Palabras Clave: Cenibroca, formaldehido, Aspergillus sp., Penicillum sp., Hypothenemus hampei.

\begin{abstract}
The use of antimicrobial agents in artificial diets for the mass rearing of insects is commonly employed to prevent the proliferation of environmental microorganisms, preventing contamination of food and space of the rear insects. Therefore, the aim of this research was to evaluate the effect of formaldehyde and nipagine antimicrobials on the Cenibroca artificial diet for coffee berry borer breeding. Through this experiment, using the Cenibroca diet, the largest number of coffee borer biological states per $\mathrm{cm} 3$ of diet was achieved, however the highest percentage of contamination by the fungi Aspergillus sp. and Penicillum sp. was obtained. When $2.65 \mathrm{~mL}$ of formaldehyde per liter of Cenibroca were added, the least diet contamination (3.3\%) was achieved. This addition did not affect the production of biological states of the coffee borer, in comparison with the rest

${ }^{1 *}$ Profesor Titular, Facultad de Ciencias Agrícolas, Programa de Ingeniería Agronómica, Universidad de Nariño, Torobajo, Pasto, Colombia. Correo electrónico: titobacca@gmail.com.

2Investigador Científico III, Disciplina de Entomología, Centro Nacional de Investigaciones de Café: CENICAFÉ, Chinchiná, Caldas, Colombia. Correo electrónico: pablo.benavides@cafedecolombia.com.co.
\end{abstract}


Bacca y Benavides - Anticontaminantes para dieta de broca del café

of diets used, in which other antimicrobial agents were used. Based on these results, it is possible to use the Cenibroca diet in coffee berry borer breeding for the mass production of its parasitoids.

Key-words: Cenibroca, formaldehyde, Aspergillus sp., Penicillum sp., Hypothenemus hampei.

\section{INTRODUCCIÓN}

La cría de insectos en el laboratorio es una práctica comúnmente utilizada en la entomología aplicada, teniendo como finalidad la disponibilidad continua de insectos. Éstas son utilizados principalmente para la cría de enemigos naturales de plagas, en programas como la técnica del insecto estéril (Parra 2012). En las cría de insectos, uno de los mayores problemas es la contaminación ocasionada por microorganismos en la dieta artificial, éstos impiden el normal desarrollo de los insectos debido a que las toxinas producidas por estos contaminantes, afectan el desarrollo de los insectos, además de cambiar la composición química de algunos ingredientes de la dieta (Cohen 2004). Estos microorganismos generalmente son hongos como; Aspergillus niger, Aspergillus flavus, Rhizopusnigricans, Cladosporium sp., Fusarium sp. y varias especies de bacterias y levaduras (Cohen 2004). Estas contaminaciones se originan desde la manipulación y los ingredientes de la dieta, los equipos, los contenedores y los insectos utilizados en la cría (Sikorowski y Lawrence 1994; Parra 2012). Existen varias estrategias para disminuir estos contaminantes y todo inicia con la asepsia de todos los operarios, instalaciones, equipos y materiales utilizados. En la asepsia se incluyen la utilización de la esterilización con autoclave y utilización de desinfectantes como el hipoclorito de sodio. Otra forma para evitar la contaminación es mediante la utilización de químicos antimicrobiales que son mezclados en la dieta. Algunos de los compuestos más utilizados son; metilparabeno, formalina, ácido benzoico, propilparabeno, ácido sorbico, ácido propiónico entre otros productos (Cohen 2004; Parra 2012).

Según Gast (1969) para evitar las contaminaciones de las crías de insectos como Colepotera y Lepidoptera, el pH de la dieta debe estar cercano a 7, por lo tanto, la escogencia de algún agente antimicrobial, como los acidulantes se deben tener en cuenta. Generalmente los productos antimicrobiales son también tóxicos para los insectos, por tanto los productos a utilizar deben ser tolerados por los insectos criados. Así mismo, su uso debe ser alterado para evitar la resistencia de los microorganismos.

La broca del café Hypothenemus hampei es la principal plaga del café en Colombia y en el mundo (Bustillo 2008). En este país el principal enemigo natural exótico de origen africano, es Prorops nasuta (Hymenotera: Bethylidae), este fue el único parasitoide introducido que se estableció exitosamente en las zonas cafeteras de Colombia y juega un papel importante en la regulación natural de la poblaciones de broca que afectan la producción de café (Maldonado y Benavides 2007). Las poblaciones de la avispa $P$. nasuta no son constantes en los cafetales, estas son disminuidas principalmente durante la época de la cosecha de café, donde son retiradas de los cafetales con los frutos brocados. Además, P. nasuta es afectada por 
las condiciones medioambientales y naturales, bajo esta condiciones es posible restaurar la poblaciones con liberaciones del parasitoide obtenidas en laboratorio, mediante la cría del hospedero de la broca del café obtenidas en dietas artificiales.

Portilla y Street (2006), proponen una dieta artificial para la cría de broca Hypothenemus hampei (Coleoptera: Curculionidae: Scolytinae) denominada Cenibroca, la cual contiene los nutrientes necesarios $y$ anticontaminantes para su cría masiva. En ensayos preliminares se pudo observar que a pesar de obtener alto número de estados biológicos de broca, se obtenían altos porcentajes de contaminación. Este hecho imposibilita su utilización para realizar crías masivas de esta plaga, cuando se piensa en adelantar un programa de cría masiva de parasitoides obtenidos a partir de broca procedente de dietas artificiales. Por lo tanto, este estudio tuvo como objetivo evaluar diferentes anticontaminantes en la dieta Cenibroca, para obtener los beneficios reportados por Portilla y Street (2006).

\section{MATERIALES Y MÉTODOS}

Este experimento se realizó en el Centro Nacional de Investigaciones de Café, Cenicafé, localizado en Chinchiná, Caldas, en los laboratorios de la disciplina de entomología. La temperatura del laboratorio fue de $25^{\circ} \mathrm{C}$ y $80 \%$ de humedad relativa. La dieta artificial para la cría de la broca del café fue realizada según lo descrito en Portilla y Street (2006), Las brocas de café fueron obtenidas del pie de cría de broca de grano pergamino, mantenidas en Cenicafé.
Los tratamientos consistieron en evaluar la dieta Cenibroca propuesta por Portilla y Street (2006) (Tabla 1) y la dieta D-200, que es una adaptación a las dietas utilizada por Villacorta (1985) y Brun et al. (1993) (Tabla 1), además de dos tratamientos que fueron la dieta Cenibroca bajo la acción del formaldehido y el nipagin mezclados y por separado, además de un tratamiento de la dieta Cenibroca más la mezcla de formaldehido y el nipagin (Tabla 2).

La dieta se sirvió en un volumen de $3 \mathrm{~mL}$ en viales de vidrio de $200 \mathrm{~mL}$ de $5 \mathrm{~cm} \mathrm{X} \mathrm{2,5} \mathrm{cm}$ de diámetro en un cámara de flujo laminar, posteriormente se llevaron a una estufa durante aproximadamente 24 horas hasta alcanzar una humedad en la dieta de 45\%. Las brocas se desinfestaron utilizando una solución hipoclorito de sodio al 3\% durante 30 segundos y se enjuagaron dos veces con agua destilada estéril, luego se secaron con papel toalla estéril. En cada tubo con dieta se depositó una broca hembra recién emergida que constituyó la unidad experimental. Cada tratamiento tuvo 30 repeticiones. El porcentaje de contaminación producida por hongos, se evaluó 30 días después de la infestación de la dieta con la broca, tiempo en el cual la broca ya ha cumplido su ciclo biológico y es posible evaluar el número de brocas producidas en la dieta en la primera generación.

Para evaluar la contaminación se tuvo en cuenta los signos de los hongos contaminantes que aparecían en la dieta, como aparición de micelios, espora y coloración diferentes a la dieta. A los 60 días después de la infestación con la broca, en cada unidad experimental se evaluó el número de estados biológicos de broca por vial que se producen en la primera 
Tabla 1. Lista de ingredientes utilizados para preparar un litro de dietas para la cría de broca del café.

\begin{tabular}{|c|c|c|}
\hline Ingredientes & $\begin{array}{c}\text { Dieta Cenibroca de Portilla y } \\
\text { Street (2006) }\end{array}$ & $\begin{array}{c}\text { Dieta D-200, adaptada de Villacorta } \\
\text { (1985) y Brun et al. (1993) }\end{array}$ \\
\hline Agar & $10 \mathrm{~g}$ & $28 \mathrm{~g}$ \\
\hline Caseína & $15 \mathrm{~g}$ & $26,6 \mathrm{~g}$ \\
\hline Levadura - Candida utilis & $15 \mathrm{~g}$ & $26,6 \mathrm{~g}$ \\
\hline Azúcar & $10 \mathrm{~g}$ & $18,6 \mathrm{~g}$ \\
\hline Café molido $12 \%$ de humedad & $30 \mathrm{~g}$ & $133 \mathrm{~g}$ \\
\hline Acido Benzoico & $1 \mathrm{~g}$ & $1 \mathrm{~g}$ \\
\hline Benomil & $1,2 \mathrm{~g}$ & $2 \mathrm{~g}$ \\
\hline Vitaminas Vanderzan & $0,5 \mathrm{~g}$ & 0,66 \\
\hline Sales de Wesson & $0,8 \mathrm{~g}$ & $2,65 \mathrm{~g}$ \\
\hline Formaldehído & $1 \mathrm{~mL}$ & $2,65 \mathrm{~mL}$ \\
\hline Etanol puro & $10 \mathrm{~mL}$ & 13,3 \\
\hline Nipagin & & $1,33 \mathrm{~g}$ \\
\hline Colesterol & & $0,8 \mathrm{~g}$ \\
\hline Ácido Ascórbico & & $0,3 \mathrm{~g}$ \\
\hline
\end{tabular}

Tabla 2. Tratamientos utilizados en la evaluación de anticontaminantes utilizados en la dieta Cenibroca, Portilla y Street (2006) y comparados con la dieta D-200.

Tratamientos

Dieta utilizada con la variación de los anticontaminantes

\begin{tabular}{ll}
\hline 1 & Dieta Cenibroca \\
2 & Dieta Cenibroca $+2,65 \mathrm{~mL}$ deformaldehido \\
3 & Dieta Cenibroca $+1,33 \mathrm{~g}$ de nipagin \\
4 & Dieta D- 200 \\
5 & Dieta Cenibroca $+2,65 \mathrm{~mL}$ Nipagin $+2,65 \mathrm{~mL}$ de formaldehido \\
\hline
\end{tabular}

y segunda generación de las brocas fundantes, mediante observaciones realizadas en el estereoscopio.

El número de estados biológicos obtenidos en cada tratamiento fueron sometidos a un análisis de varianza $(p \leq 0.05)$ y para la separación de promedios fue realizada una prueba de Duncan $(\mathrm{p} \leq 0.05)$.

\section{RESULTADOS Y DISCUSIÓN}

Según el análisis de varianza $(\mathrm{p} \leq 0.05)$ para la variable número de estados biológicos de broca, se encontraron diferencias significativas entre los tratamientos evaluados. Al realizar la prueba de Duncan $(p \leq 0.05)$, los tratamientos Cenibroca (Tratamiento 1) y el tratamiento Cenibroca más formaldehido (Tratamiento 2) fueron estadísticamente iguales y diferentes con los demás tratamientos (Tabla 3). 
Tabla 3. Número promedio de estados biológicos de broca del café después de 60 días de alimentarlos en diferentes dietas artificiales más el efecto de anticontaminantes.

\begin{tabular}{lcc}
\hline Tratamientos & N & $\begin{array}{c}\text { Promedio } \pm \text { error estándar estados } \\
\text { biológicos de broca del café/3 } \mathbf{~ c m}^{\mathbf{3}} \text { dieta }\end{array}$ \\
\hline Dieta Cenibroca & 16 & $64,88 \pm 7,40 \mathrm{~A}$ \\
Dieta Cenibroca + Formol & 29 & $57,75 \pm 10,02 \mathrm{~A}$ \\
Dieta Cenibroca + Nipagin & 27 & $35,52 \pm 5,86 \mathrm{~B}$ \\
Dieta D- 200 & 27 & $34,04 \pm 3,90 \mathrm{~B}$ \\
Dieta Cenibroca + Nipagin + Formol & 23 & $17,30 \pm 3,29 \mathrm{~B}$ \\
\hline
\end{tabular}

Al determinar el porcentaje de contaminación de las dietas a los 30 días después de haberla infestado con broca, se pudo observar que el mayor porcentaje de contaminación por los hongos Aspergillus sp. y Penicillum sp., se obtuvo en el tratamiento en la dieta Cenibroca y el menor porcentaje se obtuvo cuando a este tratamiento se adicionó formaldehido (Tabla 4). Este compuesto está mostrando algún efecto microbicida al disminuir la contaminación y al permitir que la asepsia de la dieta permita el desarrollo de la broca del café.

El efecto anticontaminante del formaldehido fue evidente, al disminuir notablemente la contaminación de las dietas, esto se pudo observar cuando fueron comparadas con las demás dietas (Tabla 3). Este efecto contribuyó con que la dieta quedará libre de microorganismos competidores y que la toxinas de los hongos interfieran en el metabolismo de los insectos. Según Cohen (2004), el formaldehido es una substancia microbicida de amplio espectro muy utilizada en los ingredientes de las dietas utilizadas para criar insectos. Sin embargo, el formaldehido es una substancia altamente tóxica para humanos y además se conoce su capacidad carcinogénica. Por lo tanto, cuando se utilice el formaldehido en dietas se deben tomar todas las medidas de precaución y protección para los operarios, además su manipulación se debe realizar en cámara extractora de gases (Cohen 2004).

El metilparabeno o nipagin es un compuesto antifúngico y tiene leve propiedades antibacteriales (Cohen 2004). La elección de los productos químicos antimicrobianos puede influir intensamente en los parámetros

Tabla 4. Porcentaje de contaminación por hongos (Aspergillus sp. y Penicillum sp.) de dietas artificiales de broca del café.

\begin{tabular}{lcc}
\hline Tratamientos & N & \% de contaminación \\
\hline Dieta Cenibroca & 30 & 46,66 \\
Dieta Cenibroca + Formol & 30 & 3,33 \\
Dieta Cenibroca + Nipagin & 30 & 10 \\
Dieta D- 200 & 30 & 10 \\
Dieta Cenibroca + Nipagin + Formol & 30 & 23,33 \\
\hline
\end{tabular}


biológicos y pueden reflejar la calidad de los insectos producidos. En la producción masiva de la chinche Lygus hesperus la utilización del formalaldehído y nipagin, pueden afectar la fisiología de los insectos criados en las dietas artificiales (Alverson y Cohen 2002), este mismo efecto fue encontrado en la cría de otros insectos (Bass et al. 1969, Singh y House 1970).

Es posible que el nipagin tenga un efecto deletéreo en la producción de estados biológicos de broca, porque fue donde se obtuvieron los menores valores del número de estados biológicos (Tabla 3).

Perez et al. (1995) compararon la dieta propuesta por Villacorta (1985) y la dieta de Brun et al. (1993), encontrando una mayor producción de número de estados biológicos de broca en la primera dieta y contaminaciones entre $10,41 \%$ a $27,27 \%$ y menor número de brocas y una contaminación de 7,29\% para la otra dieta evaluada. Esta dieta contenía mayor número de anticontaminantes como; propionato de sodio, ácido sórbico, penicilina, estreptomicina y ácido ascórbico, sin presencia de ácido benzoico y formaldehido (Brun et al. 1993).

Posiblemente la utilización de una mayor variedad de antimicrobiales condujo a una menor contaminación, pero se produjo menor número de brocas.

La utilización de antibióticos en las dietas en la cría de broca del café, pueden destruir microorganismos endosimbiontes como la proteobacteria Wolbachia, que conducen a la producción de progenies de únicamente machos (Vega et al. 2002). La utilización de funguicidas también pueden afectar el hongo Fusarium solani, que es un mutualista de la broca y es fuente de ergosterol en su dieta (Morales et al. 2000).

Portilla (1999a), comparó la dieta Cenibroca (donde utiliza $1 \mathrm{~g}$ de nipagin $y$ no utiliza formaldehido) con las dietas 140, ECOBROVILL-160 (que utilizan nipagin y formaldehido), en este estudio se registran bajas contaminaciones únicamente para Cenibroca y no se menciona contaminaciones en las otras dietas.

En el 2006 Portilla y Street, proponen la dieta artificial Cenibroca como una alternativa más eficiente y barata para la cría de la broca del café en laboratorio. A pesar de estar disponible esta dieta, en los diversos bioensayos realizados en Cenicafé se utiliza la modificación de la dieta artificial D-200, esto principalmente porque la dieta Cenibroca presenta altos niveles de contaminación. Mediante la realización de este experimento fue posible constatar el potencial de la dieta Cenibroca tal como fue publicado por Portilla (1999b) y Portila y Street (2006).

\section{CONCLUSIONES}

A partir de estos resultados es posible utilizar la dieta Cenibroca, tanto en bioensayos como para la cría de broca con fines de producción masiva de parasitoides. Sin embargo, para la escogencia de los anticontaminantes utilizados en la dietas para la cría estos insectos, se deben tener en cuenta que tengan buena eficacia antimicrobial, que no tenga efectos deleteros en la biología del insectos, costos bajos y seguros para humanos y para el ambiente. Por esta razón se plantea evaluar otros antimicrobiales que reemplacen al formaldehido. 


\section{REFERENCIAS}

Alverson, J. and Cohen, A. 2002. Effect of antifungal agents on biological fitness of Lygus hesperus Knight (Heteroptera: Miridae). Journal Economic of Entomology 95:256-260.

Bass, M. and Barnes, E. 1969. Toxicities of antimicrobial agents to white-fringed beetle larvae and the effectiveness of certain of these agents against microbial growth. Journal Economic of Entomology 62:718-719.

Brun, L., Gaudichon, V. and Wigley, P. 1993. An artificial diet for continuous rearing of the coffee berry borer Hypothenemus hampei (Ferrari) (Coleoptera: Scolytidae). Insect Science and its Applications 14(5/6):585-587.

Bustillo, P. 2008. Aspectos sobre la biología de la broca del café Hypothenemus hampei en Colombia, En: Bustillo P.

A. E. (Ed), Los Insectos y su manejo en la Caficultura Colombiana. Cenicafé, Chinchiná, Caldas, p388-418.

Cohen, A. 2004. Insect Diets: Science and Technology. CRC Press, Boca Raton.

Gast, R. 1968. Mass rearing of insects: its concept, methods and problems, En: International Atomic Energy Agency, IEAE (Ed). Radiation, Radioisotopes, and Rearing Methods in the Control of Insect Pests. Int. Ato. Energy Agency, Vienna, p59-67.
Parra, J. 2012. The evolution of artificial diets and their interactions in science and technology En: Panizzi, A. R. Parra, J.R. (Ed).Insect bioecology and nutrition for integrated pest management, CRC Press, Boca Raton FL, p51-92.

Pérez, L., Bustillo, P., Gonzalez, G. y Posada, F. 1995. Comparación de dos dietas merídicas para la cría de la broca del café, Hypothenemus hampei. Cenicafé 46(4):189-195.

Portilla, M. 1999a. Desarrollo y evaluación de una nueva dieta artificial para criar Hypothenemus hampei. Cenicafé 50(1):24-38.

Portilla, M. 1999b. Mass rearing technique for Cephalonomia stephanoderis (Hymenoptera: Bethylidae) on Hypothenemus hampei (Coleoptera: Scolytidae) developed using Cenibroca artificial diet. Revista Colombiana de Entomología 25:57-66.

Portilla, M. y Streett, D. 2006. Nuevas técnicas de producción masiva automatizada de Hypothenemus hampei sobre la dieta artificial Cenibroca modificada. Cenicafé 57:37-50.

Maldonado, L. y Benavides, M. 2007. Evaluación del establecimiento de Cephalonomia stephanoderis y Proropsnasuta, controladores de Hypothenemus hampei, en Colombia. Cenicafé 58:333-339. 
Morales, J., Rojas, M., Sittertz, H. and Saldaña,

G. 2000. Symbiotic relationship between Hypothenemus hampei (Coleoptera: Scolytidae) and Fusarium solani (Moniliales: Tuberculariaceae). Annals of the Entomological Society of America, 93(3):541-547.

Sikorowski, P. and Lawrence, A. 1994. Microbial contamination insect rearing. American Entomologist 40(4):240-253.

Singh, P. and House, H. 1970. Antimicrobials: 'safe' levels in a synthetic diet of an insect, Agriaaffinas. Journal Insect Physiology 16:1769-1782.
Vega, F., Benavides, P., Stuart, J. J and $\mathrm{O}^{\prime}$ Neil, S. 2002. Wolbachia infection in the coffee berry borer, Hypothenemus hampei (Ferrari) (Coleoptera: Scolytidae). Annals Entomological Society of America 95(3):374-378.

Villacorta, A. 1985. Dieta meridica para criação desucessivas gerações de Hypothenemus hampei (Ferrari, 1867) (Coleoptera: Scolytidae). Anaisda Sociedade Entomologica do Brasil 14(2):315-319.

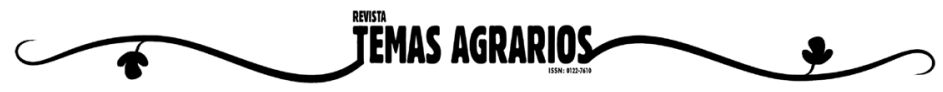

